\title{
Microbiological Comparative Studies of Crude Aqueous Extracts from Arabica and Robusta Coffee
}

\author{
Luiza-Mădălina CARACOSTEA \\ PhD Student, IOSUD Carol Davila, Bucharest, Romania \\ Rodica SîRBU \\ "Ovidius" University of Constanta, Faculty of \\ Pharmacy, Constanta, Romania \\ Anca Cristina LEPĂDATU \\ "Ovidius" University of Constanta, Faculty of \\ Natural and Agricultural Sciences, Constanta, Romania
}

\section{Abstract}

The aim of the present study was to determine the degree of microbial contamination of aqueous extracts of green and roasted coffee originating in India. The samples used in this analysis were obtained by two extraction methods: hot extraction and cold extraction. If a microbial load of samples was found, the same extracts were filtered using sterile $0.22 \mu \mathrm{m}$ pore size filters from PES (Polyester) for decontamination. The working methodology was based on the Standard SR EN ISO 21149/2017 which provides general guidelines for counting and detecting mesophilic aerobic bacteria using as a medium for isolation, cultivation and counting of microorganisms (bacteria and fungi) the non-differential environment. The experimental results obtained showed microbial contamination of all samples. The level of microbial contamination is different for the extracts taken under study, depending on the type of coffee used, green or roasted, but also on other conditions, such as wet or dry processing. Roasted coffee is much less contaminated compared to green coffee, due to a thermal (incomplete) sterilization by the roasting procedure. The working conditions used, the temperature of the distilled water used to prepare the extracts, their nonsterile handling, but also the type of coffee used, are the factors that determined the microbial contamination.

Keywords: green coffee, roasted coffee, microbial contamination, aqueous extracts, Arabica, Robusta. 


\section{Introduction}

Coffee is one of the most consumed beverages in the world, praised for its pleasant aroma, and its pharmacological characteristics. Recently, scientific and popular interest in its importance to health has increased due to the beneficial pharmacological properties established in clinical and epidemiological studies. The history of coffee dates back to about 900 A.D., when it was first mentioned by the Rhazes [Ukers W.H., 1948]. The coffee factory was originally from Ethiopia; from there it spread to Arabia, India, Ceylon, Java, Martinique, Suriname, Brazil, the Philippines and Mexico [Ukers W.H., 1948]. The genus Coffea belongs to the Rubiaceae family and includes up to 124 species. The most famous and used species are Coffea Arabica L. and Coffea Robusta L. Coffea Arabica (known as Arabica) and Coffea Canephora (known as Robusta), which represent $70 \%$ and $30 \%$, respectively in global production. [Perrois C, 2015]. Coffee cherries are treated by one of two postharvest processing methods [Silva C. F, 2008]. In Central America, Colombia and Hawaii, "wet" processing is commonly used for Arabica coffee. In the moist process, the ripe cherries are selected carefully, the pulp is mechanically separated and the fermentation follows for about 24-48 hours to remove the mucilage layer. The wet process requires more care and investment, but results in superior coffee quality [Ghosh P, 2014]. The dry method is the oldest and cheapest method of processing coffee and results in so-called natural or unspoken coffee. The dry process is preferred in countries where rainfall is scarce and extended periods of sunshine can dry the coffee properly [Silva C. F, 2008]. This method is used for about $95 \%$ of Arabica coffee made in Brazil, most of the coffee produced in Haiti, Paraguay, Ethiopia, Indonesia and some Arabica coffee made in Ecuador and India. In the dry method, the fermentation of whole fruits is essential and produces less aromatic, sweet, complex and heavy coffee in the body. The coffee fruits are placed on the ground under the sun in layers about $10 \mathrm{~cm}$ thick, piled up at night and spread every day to produce green coffee beans [Ghosh P, 2014]. The duration of sun drying lasts about 10-25 days. At that time, natural microbial fermentation takes place which can change the final quality of the coffee product. Fermentation of sugars generates ethanol and carboxylic acids. Endophytes involved in dry fermentation have a greater variety and complexity than those found during wet processing, but the real role of each group of microbiota during the fermentation of coffee by natural processing remains unknown [Silva C. F, 2008]. In this article we aim to determine the degree of microbial contamination of aqueous extracts from green coffee and roasted coffee of Arabica and Robusta species. This determination is an important analysis because the degree of contamination can be due to several factors such as: harvesting, method of processing, storage, handling, type of plant product, species used, processing and obtaining a plant extract that involves certain conditions and manipulation. We made this determination because it must be confirmed that, in order to ensure the quality of a plant extract, it is recommended to perform an adequate analysis of the microbiological risk. This determination will be useful in subsequent experiments and for testing antimicrobial 
activity but also for the use of aqueous extracts analyzed in pharmaceutical composites for topical applications.

\section{Material and Methods}

\section{Plant material}

Samples of Arabica coffee of Indian origin and Robusta coffee of Indian origin were purchased from supplier G. Bijdendijk BV, Netherlands. Plant material used was divided into 2 categories: green coffee and roasted coffee.

Green coffee beans were coarsely ground with a grinder professional coffee DIP Grinders DK-30;

The same batch of green coffee was roasted with a wood-fired roaster, which provides a increased control over the frying of the grains, ensuring that they are fried evenly, without being burned. The degree of frying was medium (at $209{ }^{\circ} \mathrm{C}$ ), and the grains have were ground with the same grinder, in the form of "fine" powder.

\section{Water-soluble coffee extracts}

The aqueous extracts were obtained from Arabica and Robusta green coffee beans but also from Arabica and Robusta roasted coffee beans. From the examined material, ground "coarse" and "fine", two types of aqueous extracts were prepared. In the first extraction method we used hot water $\left(100^{\circ} \mathrm{C}\right)$, and in the second method cold distilled water $\left(2-8{ }^{\circ} \mathrm{C}\right)$.

The extraction with hot water was performed for the Arabica and Robusta roasted coffee sample in the form of fine powder. Each sample was extracted with $100^{\circ} \mathrm{C}$ hot distilled water for 5 minutes. The ratio of roasted coffee powder to solvent was about $1: 20$.

How to work: 6 grams of Arabica and Robusta roasted coffee powder were extracted in boiling water distilled at $100^{\circ} \mathrm{C}$ for 5 minutes. After completion of the extraction operation, the extract obtained is separated from the roasted coffee residue by filter paper to remove insoluble particles in solution. The aqueous extract obtained with hot distilled water was named CA0 and CR0, respectively.

Maceration was the second process used for extraction. We used "coarse" ground green coffee and added the solvent in a tightly closed vessel and stored the bottle at $2-8^{\circ} \mathrm{C}$ for 24 hours. Water quality plays an important role in the preparation of coffee beverages, so we used distilled water, freshly prepared for all samples, during the extraction procedure. The ratio of ground coffee to solvent was 1:10. The aqueous extract obtained with cold distilled water was named CA2 and CR2, respectively. After completion of the maceration operation, the coffee extract solutions are separated from the residue by filter paper to remove insoluble particles from the solution. 


\section{Determination of the degree of microbial contamination of aqueous extracts of green coffee (CA2, CR2) and roasted (CA0, CR0)}

The materials used in this study to detect the degree of microbial contamination are represented by green and roasted coffee extracts: CA0, CA2, CR0, CR2. If a microbial load of samples was found, the same extracts were filtered using sterile $0.22 \mu \mathrm{m}$ pore size filters from PES (Polyester) for decontamination for use in subsequent experiments. The working methodology was based on the Standard SR EN ISO 21149 [SR EN ISO 21149/2017] which provides general guidelines for the counting and detection of mesophilic aerobic bacteria in cosmetics, using as a medium for isolation, cultivation and counting of microorganisms (bacteria and fungi) the non-differential environment TSA (Tryptic Soy Agar) whose technical formula is in Table 1.

Preparation of TSA medium: The components $(40 \mathrm{~g})$ are dissolved in water, the $\mathrm{pH}$ is adjusted if necessary so that after sterilization it is $7.3 \pm 0.2$ at $25^{\circ} \mathrm{C}$. The medium is distributed in test tubes in a volume of $12-15 \mathrm{~mL}$ or in vials with a maximum capacity of $500 \mathrm{~mL}$. Autoclave at $121^{\circ} \mathrm{C}$ for 15 minutes. It can be stored in the dark and at a temperature of $3 \pm 2^{\circ} \mathrm{C}$ for 1 month.

Table 1 The technical formula for the culture medium Tryptone soya agar (TSA)

\section{Tryptone soya agar}

\begin{tabular}{ll} 
Composition & Amount \\
\hline Tryptone & $15 \mathrm{~g}$ \\
Soy pepper & $5 \mathrm{~g}$ \\
Sodium chloride & $5 \mathrm{~g}$ \\
Agar & $15 \mathrm{~g}$ \\
Distilled water & $1000 \mathrm{~m}$ \\
\hline
\end{tabular}

\section{Procedure}

Transfer, with a sterile pipette (Fig. 1.), $1 \mathrm{~mL}$ of test extract to a Petri dish. $15-20 \mathrm{~mL}$ of TSA medium, previously cooled to $48^{\circ} \mathrm{C}$ on the water bath, is poured into each Petri dish under aseptic conditions (in the microbiological safety niche).

Carefully homogenize (Fig. 2) the inoculum with the culture medium and allow it to solidify, placing the Petri dishes on a horizontal and cool surface. The time between the distribution of the inoculum in the plate and the pouring of the medium must not exceed 45 minutes. The samples are inoculated in triplicate.

Turn the inoculated plates with the lid down and place in the thermostat at $32.5 \pm$ $2.5^{\circ} \mathrm{C}$ for $72 \pm 6$ hours.

After the specified incubation period, count the colonies on the plates using, if necessary, the colony counter. The plates containing at most 300 colonies are examined in diffused light. It is important that small colonies be included in the count, but it is essential to avoid confusing misleading particles of undissolved or 
precipitated plate material with small colonies. Extended colonies will be considered as a single colony. If less than a quarter of the plate is covered by extensions, count the colonies on the unaffected side of the plate and calculate the corresponding number for the whole plate. If more than a quarter of the plate is covered with extensive colonies, counting is not considered.

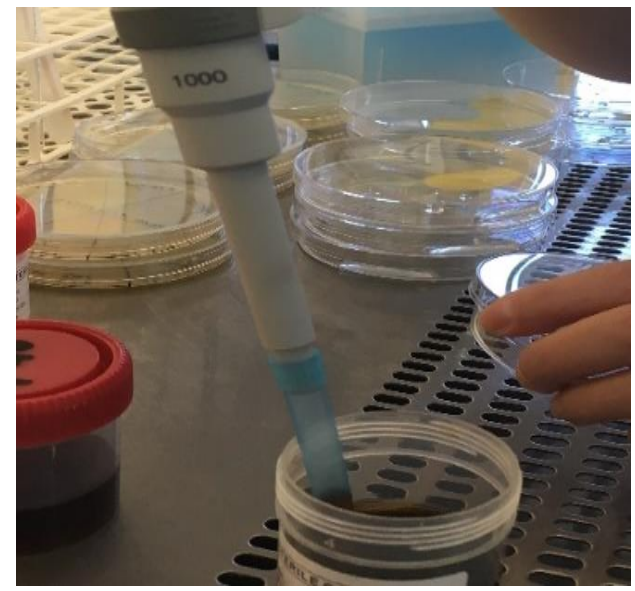

Fig. 1. Sterile transfer of test extract to Petri dishes

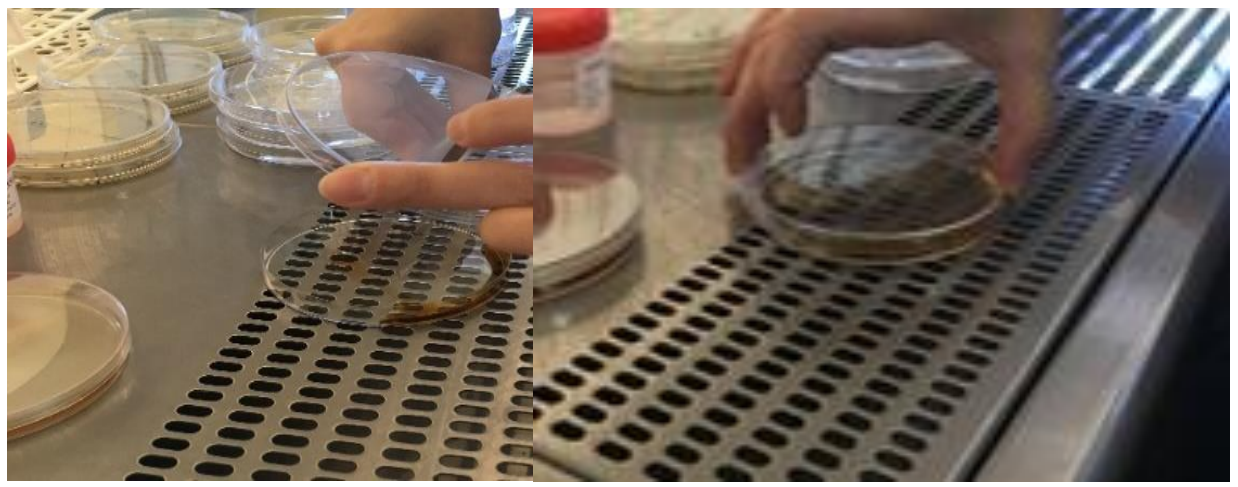

Fig. 2. Homogenization of the inoculum with the culture medium

\section{Results}

After the expiration of the incubation time, the colonies in each plate were counted, before filtration (Fig.3.A. left and right), and the results were expressed in CFU / mL, as an average of 3 counts (in triplicate) + DS (Standard Deviation), the results being detailed in Table 2. The experimental results obtained showed microbial contamination of all samples and therefore required sterilization by filtration, both in the case of CR2 sample (over $3000 \mathrm{CFU} / \mathrm{mL}$ before filtration) and CA2 (110 CFU / $\mathrm{mL}$ ), as well as in the case of aqueous coffee extract samples CR0 (2UFC / mL) and CA0 (4UFC / mL), which had a very low degree of contamination. The level of microbial contamination is different for the extracts taken in the study, depending on 
the type of coffee used, green or roasted, but also on other conditions, such as wet or dry processing. Roasted coffee is much less contaminated compared to green coffee, due to a thermal (incomplete) sterilization by the roasting procedure. The working conditions used, the temperature of the distilled water used to prepare the extracts, their non-sterile handling, but also the type of coffee used, are the factors that determined the microbial contamination. Since the sterilization of the extracts by filtration was efficient, after filtration no microbial colonies appeared on the culture medium (Fig. 3.B., left and right).

Table 2. Microbial load of coffee extracts

\begin{tabular}{|l|l|l|}
\hline $\begin{array}{l}\text { Code } \\
\text { Extract }\end{array}$ & $\begin{array}{l}\text { Microbial load (CFU / mL) } \\
\text { before filtration, expressed as a } \\
\text { mean of 3 counts + DS }\end{array}$ & $\begin{array}{l}\text { Microbial load (CFU / mL) after } \\
\text { filter sterilization }\end{array}$ \\
\hline CA0 & $4+3,7$ & 0 \\
\hline CR0 & $2+2,5$ & 0 \\
\hline CA2 & $110+2,3$ & 0 \\
\hline CR2 & $>3000$ & 0 \\
\hline
\end{tabular}

Legend: DS = standard deviation; UFC = no. Colony Forming Units
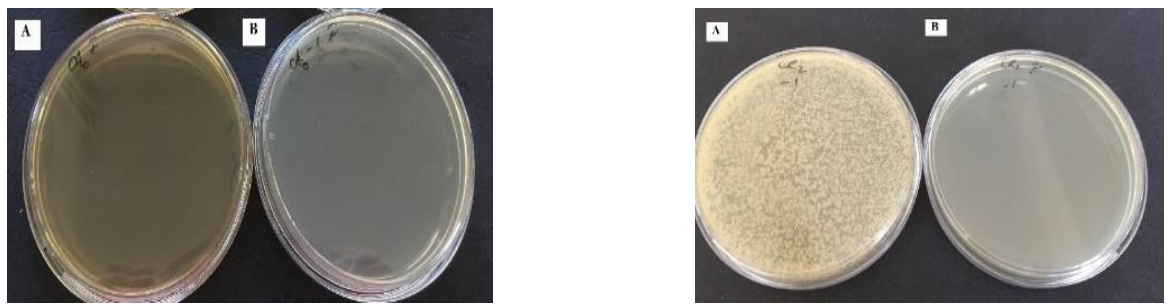

Fig.3. Extracts CA0 (left) and CR2 (right) before (A) and after (B) filtration

Green coffee beans are the final product after harvesting, washing and drying the coffee, and then roasted before grinding [Vega F. E, 2005]. Roasting seems to reduce the microbial load of CA0, CR0 coffee extracts, but does not completely eliminate it. Data from the literature indicate a $1 / 3$ reduction in the level of contamination of roasted coffee beans compared to green coffee beans [Van der Stegen GHD, 2001].

In addition to abiotic factors, such as temperature, rainfall, cultivation practices or soil changes, microbial relationships [Berg G., 2006] can be governed by the microorganisms that make up communities during fruit ripening, fermentation and drying. At the beginning of the natural processing of coffee, simple sugars such as glucose, sucrose and fructose can be used, reducing the availability of sugars for other microorganisms. Other processes, such as cultivation practices, could have influenced the diversity of microorganisms. Environmental pollution, the use of agrochemicals and intensive soil cultivation have been shown to decrease the variety of endophytic bacteria [Mekete T, 2009]. 


\section{Discussions}

Endophytic bacteria are defined as those bacteria that can be found on the surface sterilized tissue of living or isolated plants inside the plant and that do not produce any apparently harmful effect on the plant [9]. They are ubiquitous in plant tissues and have been extracted from flowers, stems, fruits, seeds, leaves, roots and root nodules. Mekete et al. [Mekete T, 2009] identified a wide variety of different genera and species of endophytic bacteria from Ethiopian coffee roots. Pseudomonas has been reported as the predominant species. The next most predominant endophyte was Bacillus. Thus, it is known that a great diversity of microbes live in coffee plants, which can be essential for the influx of coffee plant microorganisms specific to the ecosystem.

Coffee also contains a great variety of compounds which could be responsible for its antimicrobial activity. Several natural constituents of coffee have shown antimicrobial activity, among them caffeine, phenolic acids and trigonelline; however, products formed during coffee processing also present antimicrobial activity like thermal degradation products (nicotinic acid) and also products from Maillard reaction (melanoidins, alpha-dicarbonyl compounds), among others [Almeida A. A. P, 2004]. Antimicrobial agents have been widely used in the treatment of infectious diseases and in the food and cosmetics industry as antibiotics and preservatives, to effectively control microbial contamination and its consequences on the health of living organisms and the quality of a product, although the use of these chemicals antibiotics has led to many ecological and medical difficulties, due to their continuous toxicity, hormonal imbalance caused in host organisms, carcinogenic and teratogenic effects, etc.

The pathogens of infectious diseases have become resistant to antibiotics, which is why it has become a priority and strategic research of plant extracts with antimicrobial activity, in order to discover new biologically active substances with antimicrobial action. The natural antimicrobial capacity of plant extracts has been the basis of many applications, including raw materials and the preservation of processed foods, pharmaceuticals, cosmetics, in alternative medicine and natural therapies [Andrews J. M., 2005].

Following the analysis performed by us, we can appreciate that for all samples represented by aqueous extracts of green coffee (CA2 and CR2) and roasted (CA0 and CR0) the need for sterilization was imposed before they can be analyzed for testing antimicrobial activity and before introduction into semi-solid formulations for topical applications.

\section{Conclusion}

Determining the degree of microbial contamination of aqueous extracts from green coffee and roasted coffee is an important analysis because this degree of contamination can come from several factors such as: harvesting, method of 
processing, storage, handling, type of plant product, species used, processing and obtaining a plant extract that involves certain conditions of production and handling. Thus, our attempts to highlight the degree of contamination of aqueous extracts from green and roasted coffee, led us to the need to filter the samples for decontamination, especially of highly contaminated CA2 and CR2 extracts. This determination will be useful for further analyzes such as: before testing antimicrobial activity, as well as before introduction into semi-solid formulations for topical applications.

\section{References}

[1] Almeida A. A. P, Naghetini C. C, Santos V. R., Glo'ria M. B. A., In Vitro antimicrobial activity of coffee extracts on Streptococcus mutans, The 20-th International Conference on Coffee Science, Bangalore, India. Proccedings of ASIC Conferences, 2004, 242-248.

[2] Andrews J. M., BSAC standardized disc susceptibility testing method (version 4), J. Antimicrob. Chemother, 2005, 56(1), 60-76

[3] Berg G., Hallmann J., Control of plant pathogenic fungi with bacterial endophytes. In: Schulz, B., Boyle, C., Sieber, T. (Eds). Soil biology.Microbial root endophytes,.Berlin, Heidelberg, Germany, Springer, 2006, 9, 53-69

[4] Ghosh P, Venkatachalapathy N., Processing and Drying of Coffee - A review. IJERT, 2014, 3(12), 784-794

[5] Mekete T, Hallmann J, Kiewnick S, Sikora R., Endophytic bacteria from Ethiopian coffee plants and their potential to antagonise Meloidogyne incognita, Nematology, 2009, 11(1), 117-127.

[6] Silva CF, Batista LR, Abreu LM, Souza Dias E, Schwan RF., Succession of bacterial and fungal communities during natural coffee (Coffea arabica) fermentation, Food Microbiology, 2008, 25, 951-957

[7] SR EN ISO 21149 in ASRO store. Cosmetics. Microbiology. Counting and detection of mesophilic aerobic bacteria, 2017, https://magazin.asro.ro/

[8] Perrois C., Strickler S.R., Mathieu G., Leppeley M., Bedon L., Michaux l., Privat I., Differential regulation of caffeine metabolism in Coffea arabica (Arabica) and Coffea canephora (Robusta), Planta, 2015, 241, 179-191

[9] Ukers W.H., The Romance of Coffee: an outline history of coffee and coffee drinking through a thousand years. The Tea and Coffee Trade Journal Co., 1948, 40(3), 291-292

[10] Van der Stegen GHD, Essens PJM, van der Lijn J., Effect of roasting conditions on reduction of ochratoxin A in coffee, Journal of Agricultural and Food Chemistry, 2001, 49, 4713- 4715.

[11] Vega F.E, Pava, Ripoll M, Posada F, Buyer JS, Endophytic bacteria in Coffea arabica L., J. Basic Microbiol. 2005, 45, 371-380. 